Revue des patrimoines

$21 \mid 2013$

De l'art de bâtir aux champs à la ferme moderne

\title{
La ferme comme laboratoire ou l'architecture expérimentale de Charles Gilbert de Morel-Vindé (1759-1842)
}

Valérie Nègre

\section{OpenEdition}

Journals

Édition électronique

URL : http://journals.openedition.org/insitu/10347

DOI : 10.4000/insitu. 10347

ISSN : 1630-7305

Éditeur

Ministère de la Culture

\section{Référence électronique}

Valérie Nègre, «La ferme comme laboratoire ou l'architecture expérimentale de Charles Gilbert de Morel-Vindé (1759-1842) », In Situ [En ligne], 21 | 2013, mis en ligne le 12 juillet 2013, consulté le 14 novembre 2019. URL : http://journals.openedition.org/insitu/10347 ; DOI : 10.4000/insitu.10347

Ce document a été généré automatiquement le 14 novembre 2019.

\section{(c) (†) $\ominus$}

In Situ Revues des patrimoines est mis à disposition selon les termes de la licence Creative Commons Attribution - Pas d'Utilisation Commerciale - Pas de Modification 4.0 International. 


\title{
La ferme comme laboratoire ou l'architecture expérimentale de Charles Gilbert de Morel-Vindé (1759-1842)
}

\author{
Valérie Nègre
}

\section{Introduction}

1 Charles Gilbert de Morel-Vindé fait partie de ces nombreux propriétaires agronomes qui, repliés sur leurs terres pendant la Révolution, appliquent les « sciences positives " à « l'art agricole » dans le double espoir d'améliorer le rendement de leurs propriétés et de triompher de l'esprit routinier des campagnes ${ }^{1}$. Le personnage est intéressant dans la mesure où l'importance de sa fortune et sa position sociale éminente lui permettent de mettre en œuvre des idées nouvelles largement partagées par ses contemporains. Deux nous intéressent particulièrement: transformer ses propriétés «en fermes d'expériences et d'exemples $»^{2}$ et concevoir de nouveaux types de bâtiments ruraux. Ses publications sur les constructions rurales méritent l'attention car elles permettent d'observer l'émergence d'édifices dont la forme et la localisation sont déterminées par une rationalité indépendante de toute relation avec le lieu. On dirait aujourd'hui des édifices qui ne tiennent pas compte des caractéristiques environnementales, culturelles et identitaires. Ce fait est d'autant plus frappant que les constructions rurales modestes auxquelles le vicomte s'intéresse étaient traditionnellement marquées par leur localisation. C'est l'émergence de ce phénomène que l'on qualifierait aujourd'hui de « déterritorialisation » que cet article se propose d'examiner à travers quelques projets de l'agronome. La campagne, pensée comme un lieu de retards et de routines, est le terrain des expériences les plus audacieuses. L'économie de temps et d'argent, le rendement et la salubrité justifient l'importation de systèmes de distribution ou de modes de construction originaires d'autres régions ou d'autres pays. 


\section{Expérimenter d'abord}

Charles Gilbert de Morel-Vindé est conseiller au Parlement lorsque la Révolution éclate. Il échappe à l'arrestation et se retire de la vie publique jusqu'au retour de Louis XVIII qui le fait pair de France. Le baron de Frénilly le décrit comme un « parfait échappé du dix-huitième siècle » : " philosophe jusqu'aux oreilles, athée avec prosélytisme, ôtant la religion à sa femme et la refusant à ses enfants, attaché à la monarchie, ennemi de la Convention, du Directoire, de Bonaparte et ivre de la Restauration $\|^{3}$ (fig. $\mathbf{n}^{\circ} \mathbf{1}$ ). Ses biographes et ses contemporains insistent sur son caractère profondément libéral. On sait que tous ses serviteurs étaient intéressés à bien remplir ses ordres et ses intentions et "afin qu'aucun d'eux ne rêvât au plaisir de figurer sur son testament", il avait instauré des étrennes progressivement croissantes « à mesure que le maître vivait plus longtemps». Les facettes de ce richissime collectionneur ${ }^{4}$, homme d'affaires avisé, auteur de romans "pleins de grâce et de fraîcheur $\aleph^{5}$ autant que d'études statistiques minutieuses ne sauraient être résumées en quelques lignes.

Figure 1

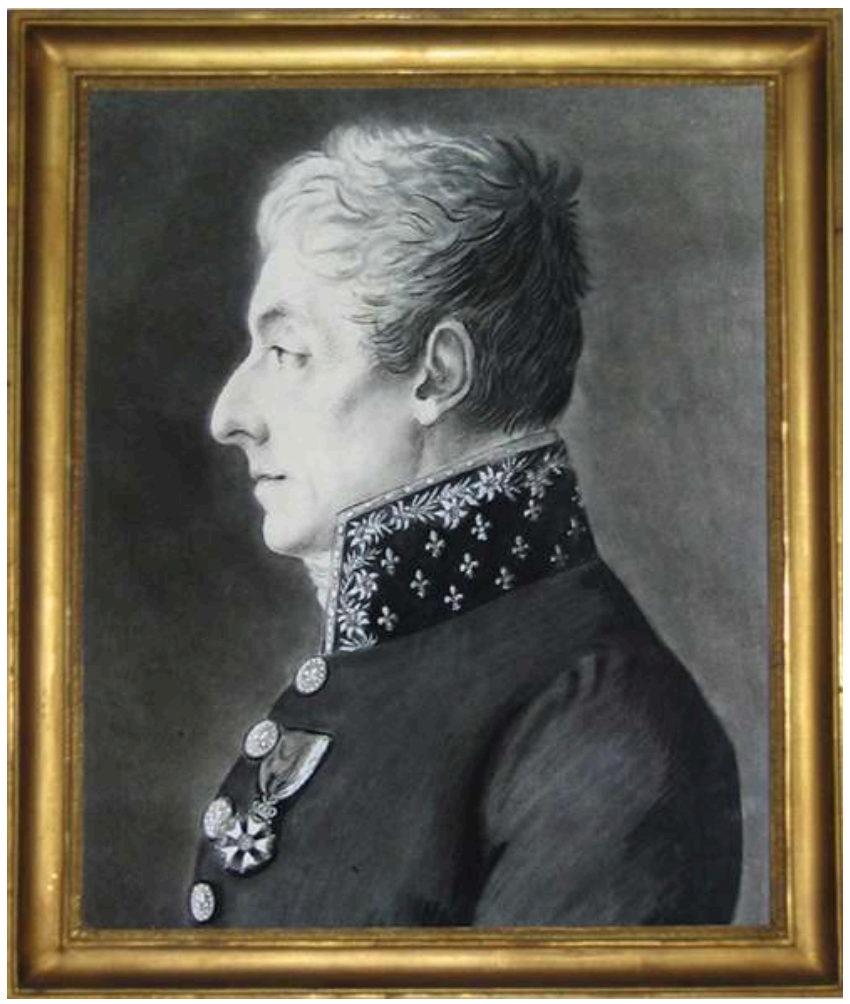

Charles Gilbert de Morel-Vindé.

Phot. Nègre, Valérie. (C) Valérie Nègre.

3 Ses activités dans le domaine de "l'économie rurale » sont en elles-mêmes multiples ${ }^{6}$. La manière dont il se passionne pour la propagation des moutons Mérinos à la laine fine et abondante et en particulier dont il traite ses troupeaux espagnols (et ses fermiers) à Versailles (1808) et en Champagne (1816) est révélatrice de son approche résolument scientifique : numérotage systématique des animaux, repérage des jours de monte et d'agnelage. Son biographe le décrit comme attaché à « vaincre la passivité de la brebis 
pour en faire une bonne amante ", mission qu'il savait difficile ayant observé avec une très grande précision ses mœurs, la sachant, d'un " très-faible degré de sensibilité dans tous les actes de sa vie [...] sans passions apparentes $»^{7}$. Les statistiques lui permettent de calculer l'âge exact auquel les brebis doivent être fécondées, établir des règles pour augmenter le nombre de naissances et détruire quelques préjugés : qu'il est faux par exemple que le bélier puisse féconder cent brebis dans vingt-quatre heures, «le plus vigoureux " n'étant capable d'en féconder que vingt-quatre tout au plus ${ }^{8}$. Sous l'œil perplexe de ses fermiers, Morel-Vindé développe les "prairies artificielles", acclimate les pins maritimes en Champagne et les fraisiers des Alpes à Versailles. Rien d'étonnant de le voir figurer parmi les quarante trois premiers souscripteurs de la célèbre « ferme modèle » de Roville (Meurthe), fondée en 1822 par Mathieu de Dombasle.

4 La contribution à l'architecture rurale de ce " grand seigneur volontairement paysan $»^{9}$ est à la fois modeste et ambitieuse. Modeste par le nombre et la taille des constructions effectivement réalisées ; ambitieuse par la volonté de mettre au point, partant de là, un type idéal de construction valable pour tous les bâtiments de la ferme et, plus encore, pour toutes les colonies agricoles à venir. Ce qui s'exprime clairement dans le double aspect de ses publications, tantôt imprimées sous forme de petites brochures in-octavo extraites de revues savantes, tantôt éditées par l'architecte Adrien Louis Lusson sous forme de recueils in-folio lithographiés au trait, destinés à un public plus large ${ }^{10}$. Son Essai sur les constructions rurales (1824) ${ }^{11}$ est ainsi repéré par le botaniste écossais John Claudius Loudon qui redessine l'ensemble et ajoute même des vues isométriques pour son Encyclopedia of Cottage, Farm, and Villa Architecture (1833) ${ }^{12}$ (fig. n' ${ }^{\circ}$ ). Cette publication explique probablement la construction d'une ferme "à la Morel-Vindé » à Bernardston dans le Massachusetts en 1833. Le recueil de Morel-Vindé trouve également des échos dans la revue périodique La maison rustique du XIXe siècle (1835) et dans le Cours complet d'agriculture (1836). Une maquette de sa ferme idéale entre dans les collections du Conservatoire des arts et métiers en 1840. 


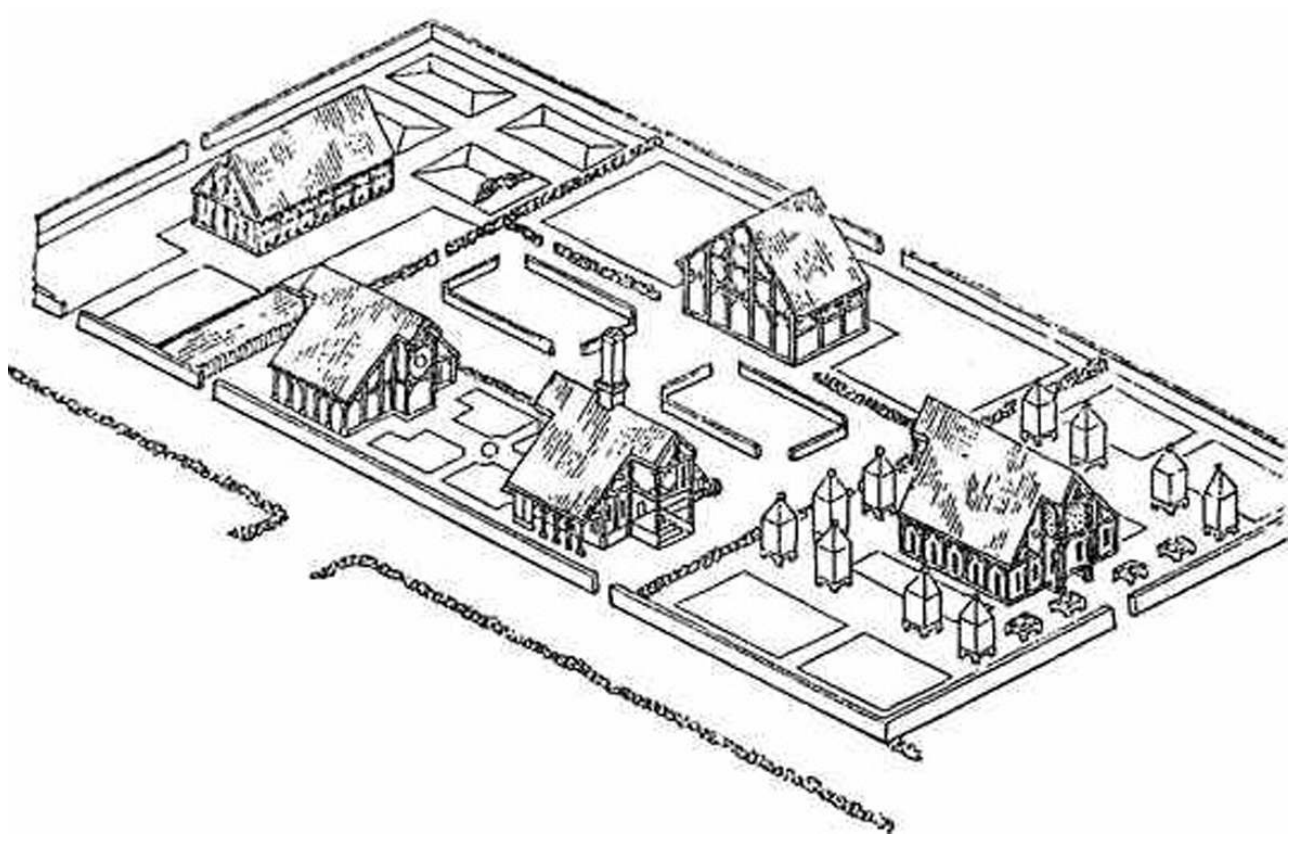

Isométrie de la ferme imaginée par Morel-Vindé d'après John Claudius Loudon. Encyclopedia of Cottage, Farm, Villa Architecture, (1833).

Repro. Nègre, Valérie. (c) Valérie Nègre.

5 Le premier principe qui guide Charles Gilbert de Morel-Vindé - l'économie - le conduit à rejeter la construction en maçonnerie et à choisir le pan de bois, considéré comme moins cher. La grange qu'il fait exécuter sans architecte, en 1812, dans son domaine de la Celle-Saint-Cloud, près de Versailles, témoigne de son approche rationaliste. La forme, les dimensions et les détails de construction sont déterminés par des considérations d'ordres économiques et fonctionnelles. L'édifice sur poteaux est relativement élevé par rapport au sol (un mètre) pour éviter que les rats et les souris n'y pénètrent ${ }^{13}$; la trame de onze pieds $(3,50 \mathrm{~m})$ correspond au « maximum de la portée des bois posés en travers $»^{14}$; les assemblages sont les plus simples (à mi-bois ou à tiers bois); la construction est totalement isolée pour que les voitures destinées à décharger les gerbes puissent l'approcher aisément et assez spacieuse pour que l'opération de battage puisse se faire à l'intérieur (fig. $\mathbf{n}^{\circ} 3$ ). 


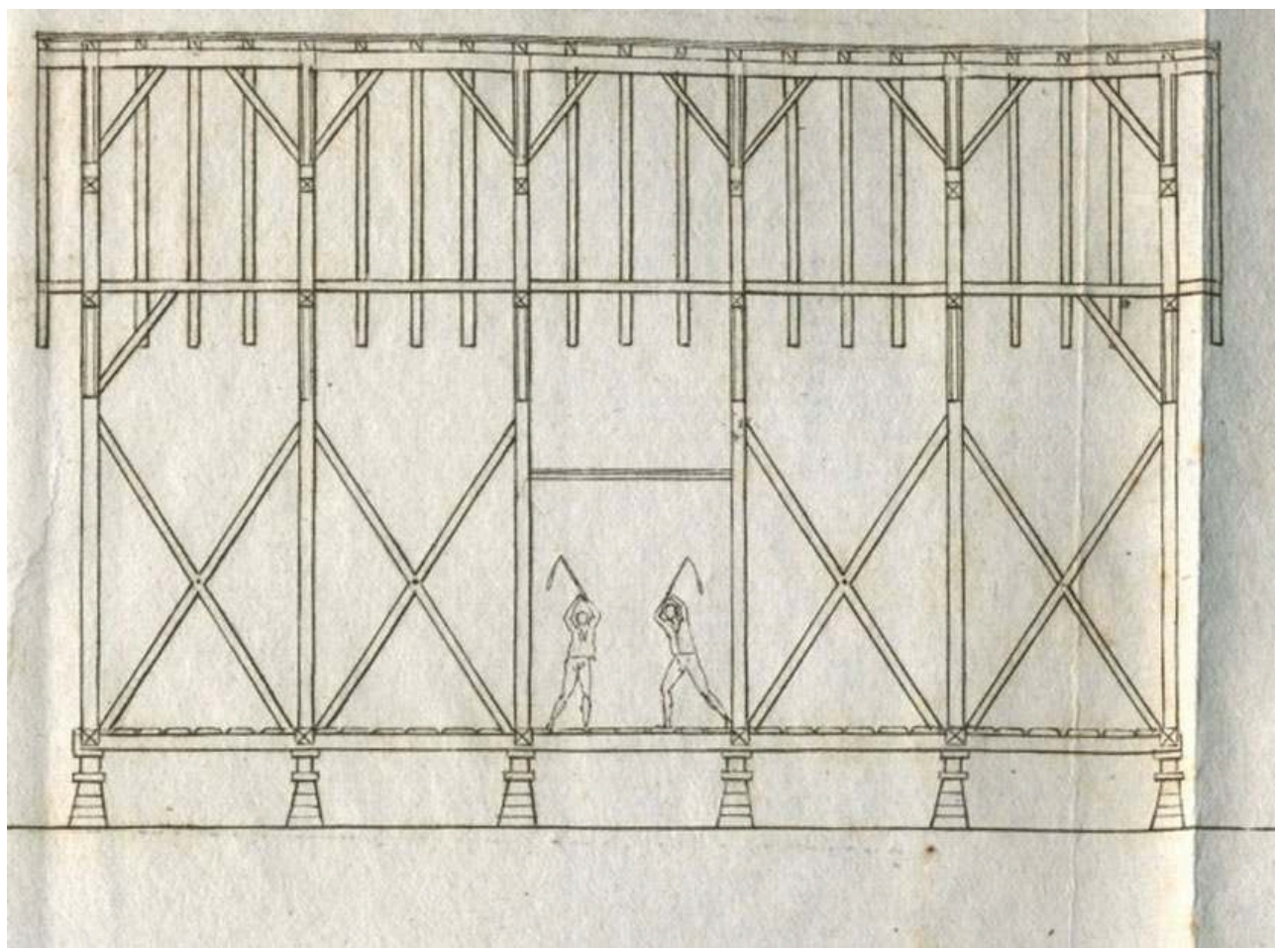

Grange construite par l'entrepreneur La Casse à la Celle-Saint-Cloud en quatre mois, de mars à juin 1812. $55 \times 22$ pieds (17,4 $\times 7 \mathrm{~m})$, d'après Plans et détails d'une nouvelle construction rurale pour servir de grange, 1813, pl. 1.

Repro. Nègre, Valérie. (C) Valérie Nègre.

6 Mis à part le marche pied mobile en fer et l'habillage des poteaux en ardoise et en verre, Morel-Vindé présente l'ensemble comme une construction ne faisant appel qu'à des «moyens connus». Ce qui a néanmoins pour effet d'attirer une "affluence de curieux $»^{15}$. En effet, trois traits au moins sont étonnants : l'emploi de pilotis pour un édifice aussi vaste, l'utilisation de bois parfaitement équarris et la couverture en ardoise. À en croire la perspective, cet ensemble parfaitement rectiligne devait avoir un aspect étonnant pour une construction agricole modeste (fig. $\left.\mathbf{n}^{\circ} \mathbf{4}\right)$. Morel-Vindé précisait que l'équarrissage à la scie, et non à la cognée, devait permettre la récupération des parties équarries (rondes en dessous et plates sur le dessus) pour le plancher, ce que la perspective ne montre pas. Pour le choix de la couverture en ardoise, en revanche, matériau plutôt coûteux, aucun argument n'était avancé; l'agronome écrivait seulement qu'elle était « plus solide, plus économique qu'en tuile et même en paille $»^{16}$. 
Figure 4

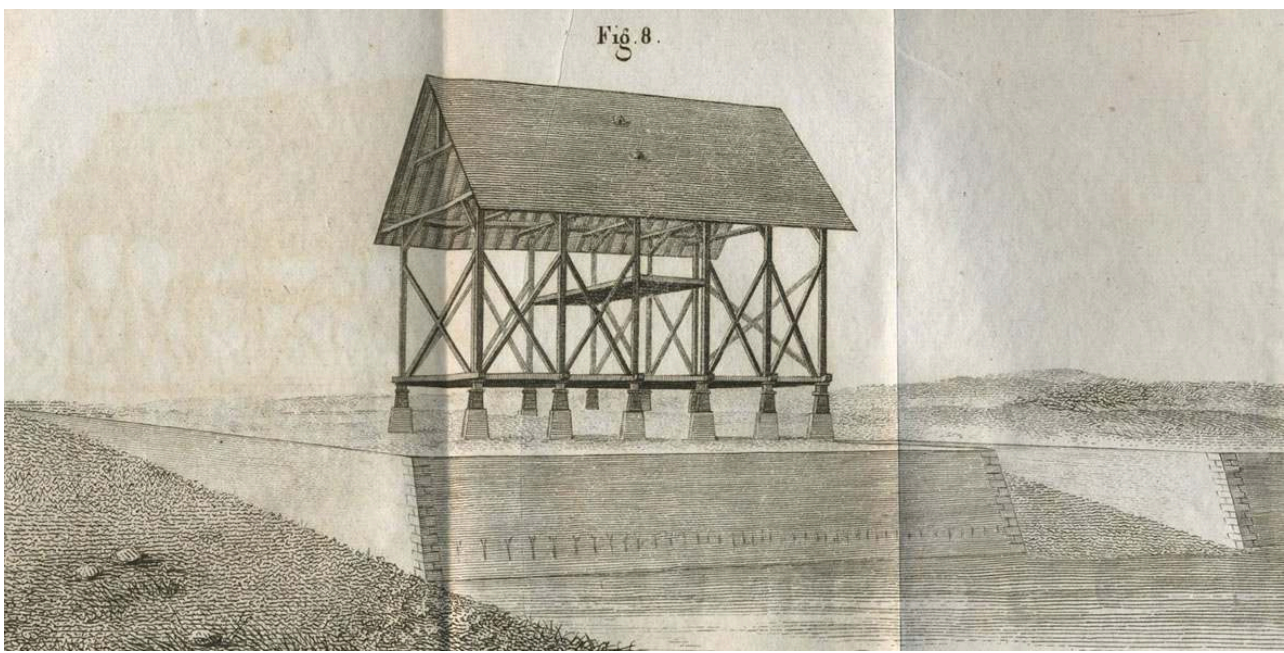

Perspective de la grange, d'après Plans et détails d'une nouvelle construction rurale pour servir de grange, 1813, pl. 2.

Repro. Nègre, Valérie. @ Valérie Nègre.

7 La bergerie construite quelques temps après à la Celle-Saint-Cloud, toujours sans architecte, poursuit le même objectif : « servir de modèle de la meilleure bergerie faite au plus bas prix possible $»^{17}$. Il choisit pour cela de n'employer que des bois de courtes dimensions (fig. $\mathbf{n}^{\circ}$ ). Outre l'économie, le vicomte est encore guidé par la taille des brebis. Chaque brebis est évaluée à six pieds de superficie pour les adultes, dix pour chaque portière et son agneau, ce qui permet de fixer la taille des travées qui correspond à trente brebis avec agneau ou cinquante adultes sans agneaux.

Figure 5

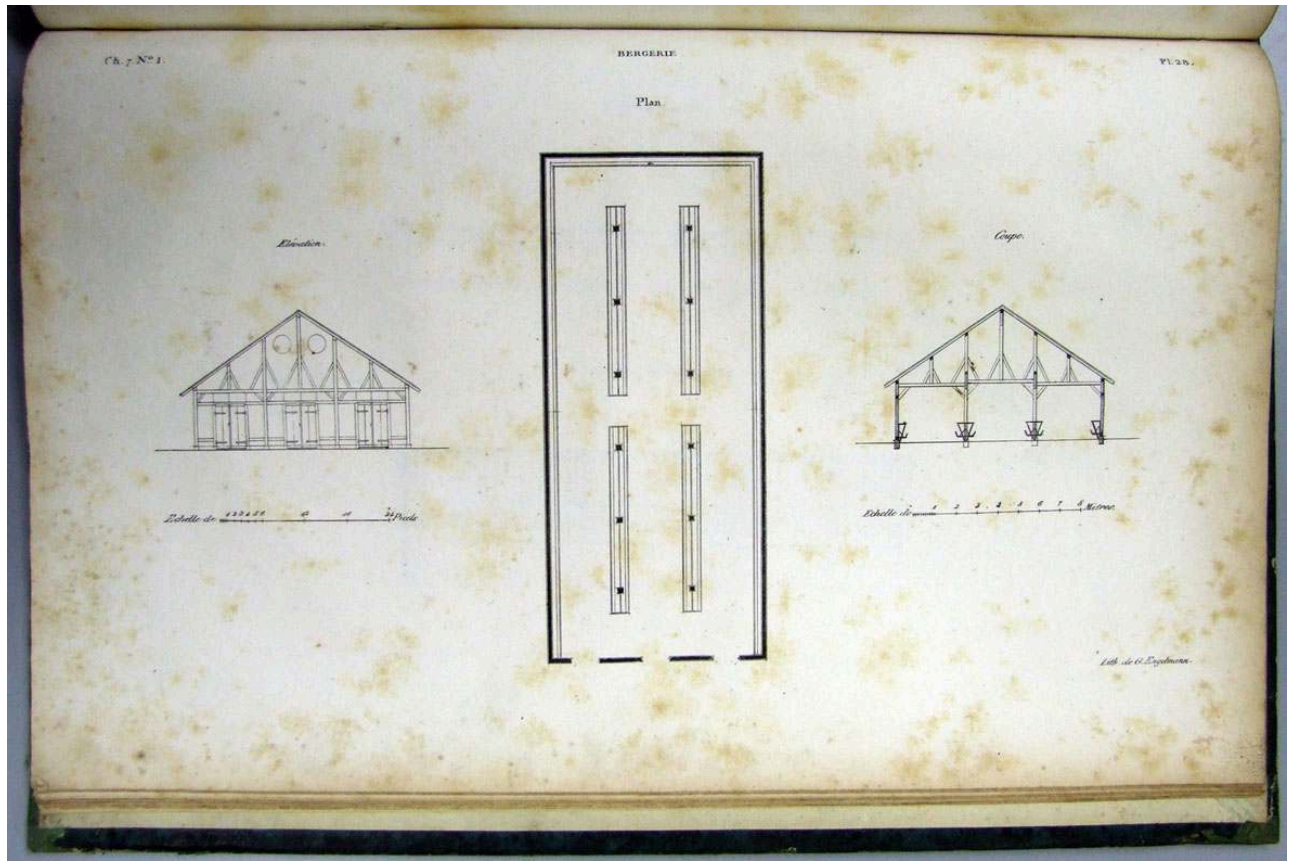

Élévation, plan et coupe de la bergerie 70 × 30 pieds $(22,2 \times 9,5$ m), d'après Plan, coupe, élévation et détails d'une bergerie, 1819.

Repro. Nègre, Valérie. (c) Valérie Nègre. 
Une construction rurale bien plus modeste encore montre le rôle déterminant qu'occupent les modèles étrangers dans les réflexions de l'agronome. Il s'agit d'un gerbier "à toit mobile", exécuté en $1811^{18}$. Les gerbiers ou les meules destinés à stocker le foin ou la paille, qui nous semblent aujourd'hui à peine relever de l'architecture, intéressaient tout particulièrement les propriétaires terriens au début du XIXe siècle, car il s'agissait de structures moins coûteuses que les granges. Le gerbier tenait selon le vicomte à la fois des meules «à l'anglaise » (cadre en bois surélevé du sol) et « à la hollandaise » (dotées de toits mobiles portées par des poteaux) (fig. $\left.\mathbf{n}^{\circ} \mathbf{6}\right)$. Il est intéressant de remarquer qu'en 1824, Morel-Vindé, avait changé d'avis à propos de ce gerbier. Les meules « à l'anglaise » et « à la hollandaise » étant jugées trop coûteuses, le vicomte préconisait alors des structures beaucoup plus simples, des «meules à terre » simplement établies sur un sol dur pilonné et couvert de fagots. Ce qui confirme ce que l'on devine, le principe d'économie était loin d'être le seul moteur de ces expériences.

Figure 6

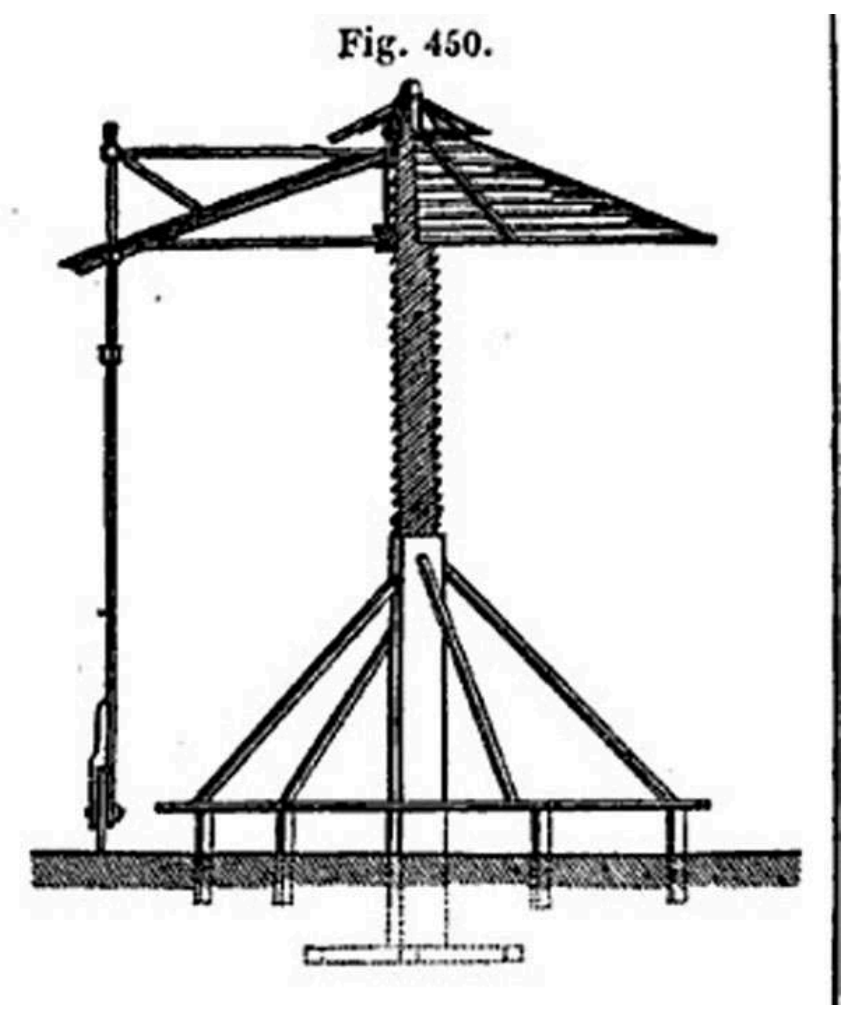

Gerbier «à toit mobile » imaginé par Morel-Vindé, d'après Jacques Alexandre Bixio, Maison rustique du XIXe siècle, p. 313, vol. 1, 1835, fig. 450.

Repro. Nègre, Valérie. (c) Valérie Nègre.

\section{Modéliser la ferme}

L'Essai sur les constructions rurales édité en 1824 par l'architecte Adrien Louis Lusson ${ }^{19}$ présente une systématisation du système de construction de la bergerie à tous les bâtiments de la ferme dans l'objectif de fournir un modèle aux colonies agricoles en $\operatorname{projet}^{20}$. La démarche est la même: faire «le mieux possible, au meilleur marché 
possible " en cherchant cette fois «les meilleures conditions communes » à tous les bâtiments ${ }^{21}$. La "solution » réside dans le système constructif : «choisir les matériaux les plus communs et les moins chers, puis mettre leur emploi à la portée des ouvriers les moins habiles de nos campagnes » et plus précisément « l'emploi général et exclusif du bois pris dans ses moindres dimensions $»^{22}$. Morel-Vindé généralise la construction à petits bois $(3,25 \mathrm{~m})$ " pas plus chers que le bois à brûler» expérimentée dans sa bergerie.

Figure 7

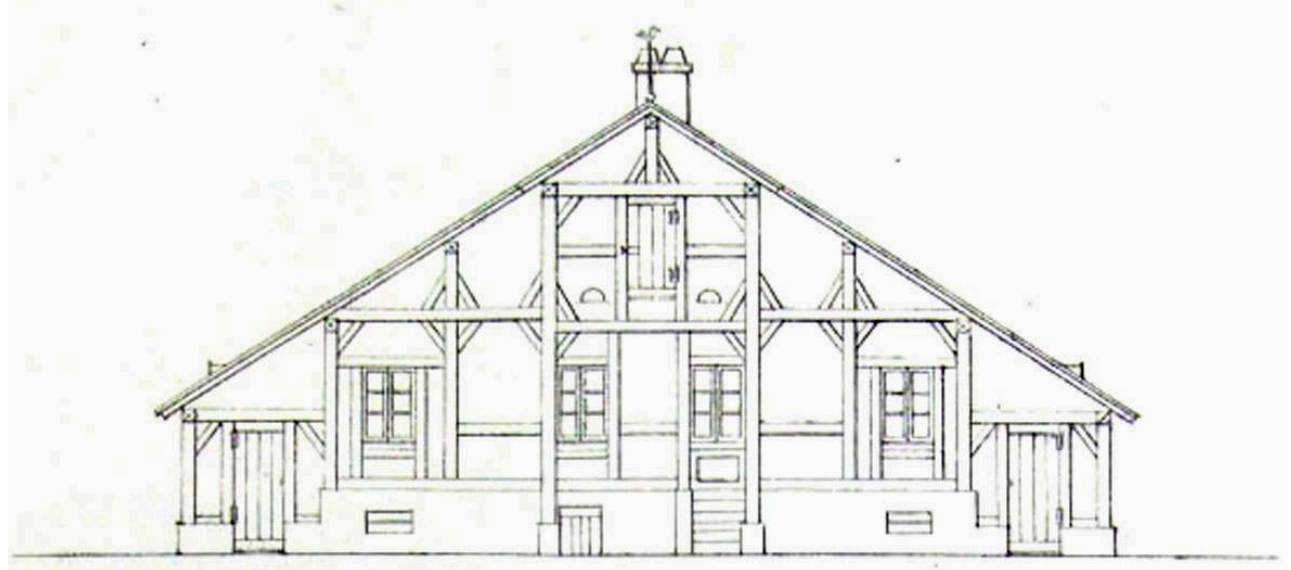

"Maison d'un journalier », d'après Essais sur les constructions rurales, 1824, pl. 2 et 4.

Repro. Nègre, Valérie. (c) Valérie Nègre.

Figure 8

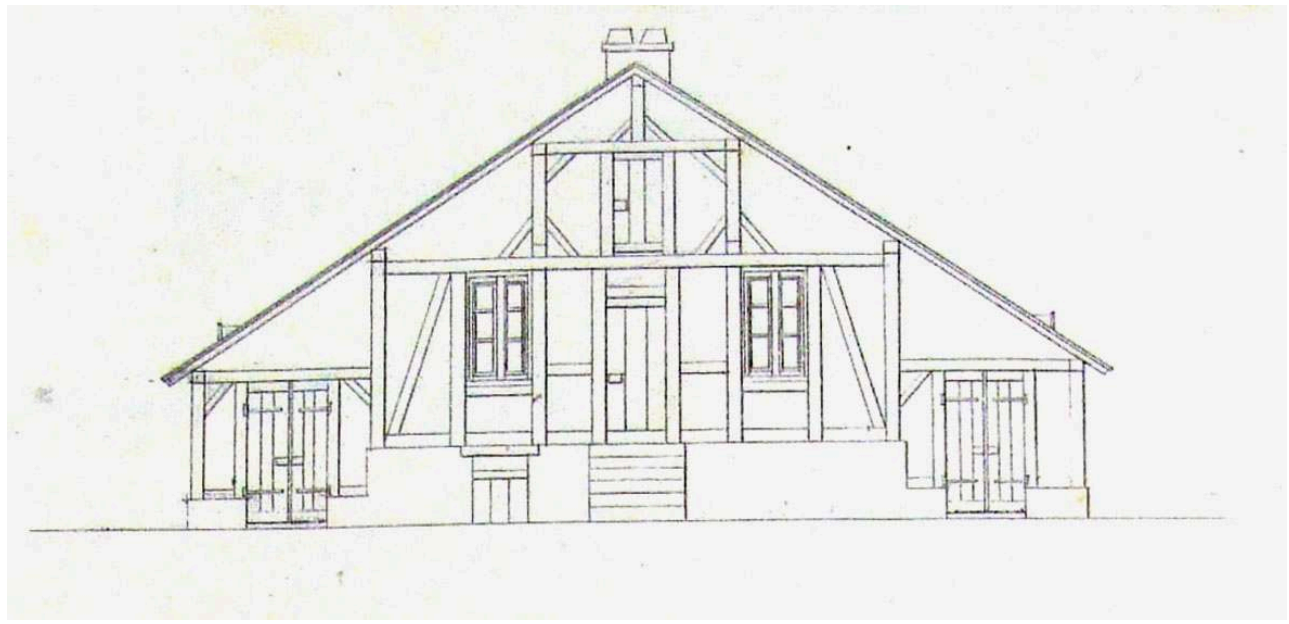

"Maison d'un petit propriétaire ou tenancier », d'après Essais sur les constructions rurales, 1824, pl. 2 et

Repro. Nègre, Valérie. @ Valérie Nègre.

Le principe n'est pas nouveau au début du XIX siècle, mais son application à l'ensemble des édifices de la ferme, de l'habitation du «plus pauvre habitant de la campagne » (le journalier), à celle du riche fermier est étonnante (fig. $\mathbf{n}^{\circ} 7$ ) (fig. $\left.\mathbf{n}^{\circ} \mathbf{8}\right)$. L'agronome pousse loin la notion de " travée ». La structure poteau-poutre le conduit à disposer des 
poteaux au milieu des pièces ${ }^{23}\left(\right.$ fig. $\left.\mathbf{n}^{\circ} \mathbf{9}\right)$ et à mettre en avant la notion de flexibilité : « les bâtiments que l'on fait ainsi peuvent s'étendre ou se restreindre à volonté et sans rien déranger à l'ensemble : ce ne sont jamais qu'une ou plusieurs travées à rajouter ou à retrancher, sans que tout le reste du bâtiment en souffre le moins du monde, et comme chacune des travées est pareille à l'autre et du même prix, à chaque tranche de bâtiments que l'on veut de plus ou de moins, on sait d'avance le surcroît ou la diminution de dépense qui en résultera $»^{24}$.

Figure 9

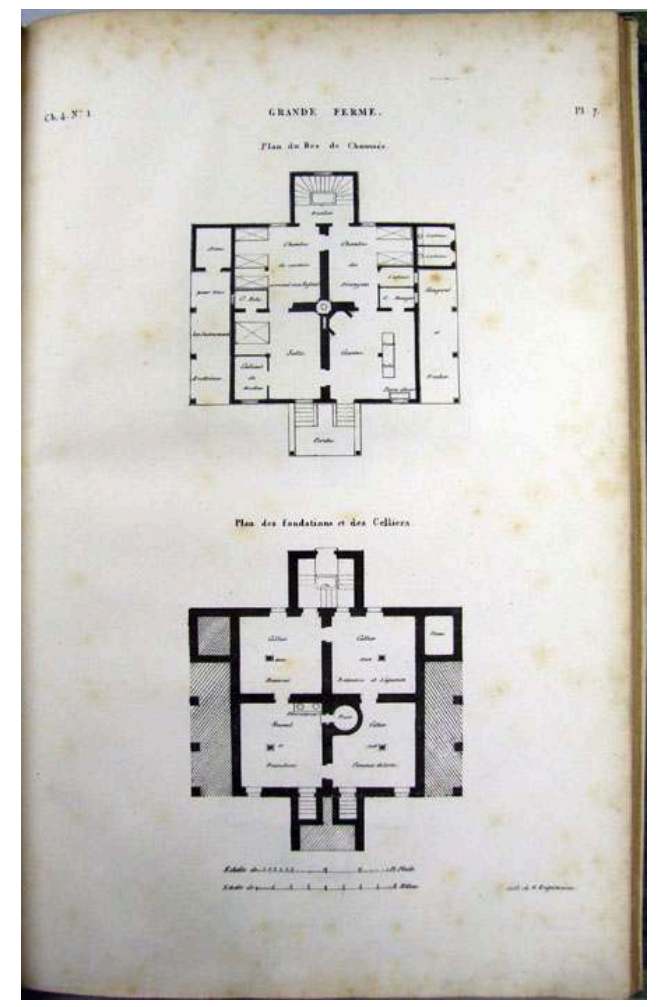

Plan de la maison d'un petit propriétaire, d'après Essais sur les constructions rurales, 1824, pl. 7.

Repro. Nègre, Valérie. @ Valérie Nègre.

11 La ferme est une juxtaposition de modèles français et étrangers. Le plan d'ensemble s'inspire de la ferme cauchoise : un espace libre quadrangulaire à l'intérieur duquel les bâtiments sont disposés librement (fig. $\mathbf{n}^{\circ} \mathbf{1 0}$ ). Le bâtiment le plus volumineux rappelle la "grange-étable limousine »: de part et d'autre d'un vaisseau central qui sert d'aire de battage et de grange, deux vaisseaux latéraux servent à loger les vaches et les chevaux. Ici, Morel-Vindé ajoute d'autres constructions (fig. $\left.\mathbf{n}^{\circ} \mathbf{1 1}\right)$ (fig. $\mathbf{n}^{\circ} \mathbf{1 2}$ ). L'ensemble forme un bâtiment mixte qui regroupe un grand nombre de fonctions. Le chauffage des habitations reprend un dispositif employé dans le nord de l'Europe : un poêle central dont le conduit est pris dans l'épaisseur du mur chauffe l'ensemble du bâtiment. 
Figure 10

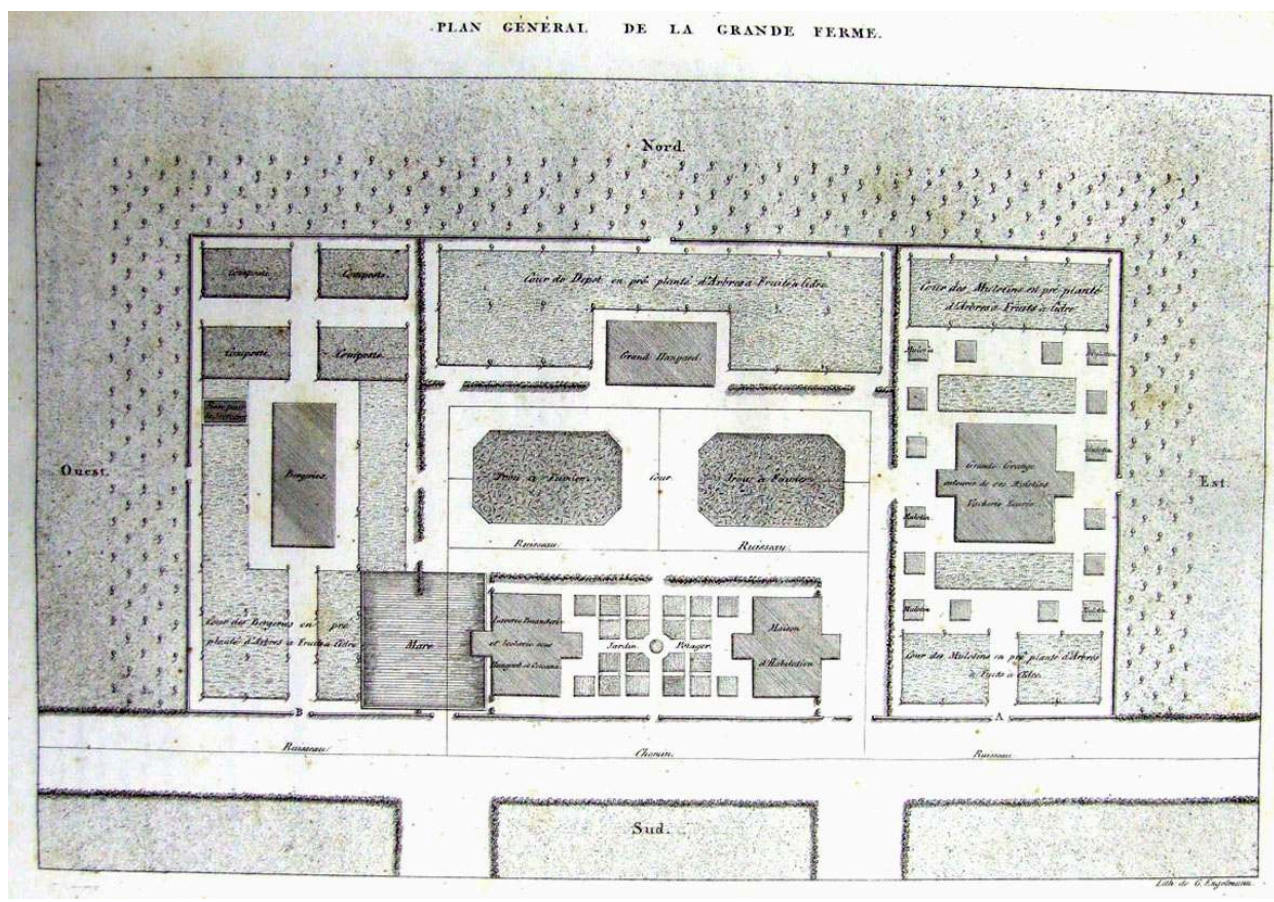

«Plan général de la grande ferme » d'après Essais sur les constructions rurales, 1824, pl. 32. La ferme est entourée d'un mur et divisée en quatre parties, chaque partie étant séparée par des fossés ou des haies pour mettre les édifices à l'abri de l'incendie et séparer les différents animaux.

Repro. Nègre, Valérie. (c) Valérie Nègre. 
Figure 11

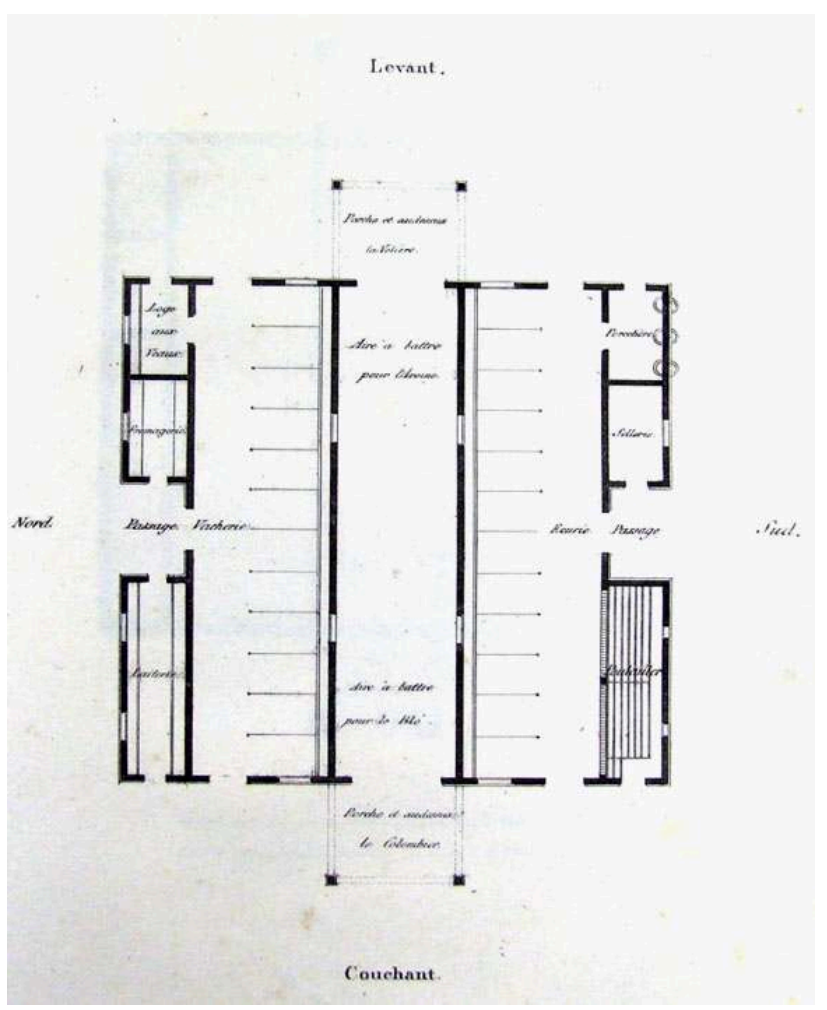

Plan de la « Grande ferme », d'après Essais sur les constructions rurales (1824), pl. 12. La grange, au centre, dotée d'une aire à battre le blé et l'avoine est encadrée par une vacherie et une écurie de douze bêtes. Au-delà, dans les parties extérieures, sont situés d'un côté la laiterie, la fromagerie et la loge pour les veaux et de l'autre le poulailler, la sellerie et la porcherie. Le colombier et la volière sont placés au-dessus du porche d'entrée.

Repro. Nègre, Valérie. (c) Valérie Nègre.

Figure 12

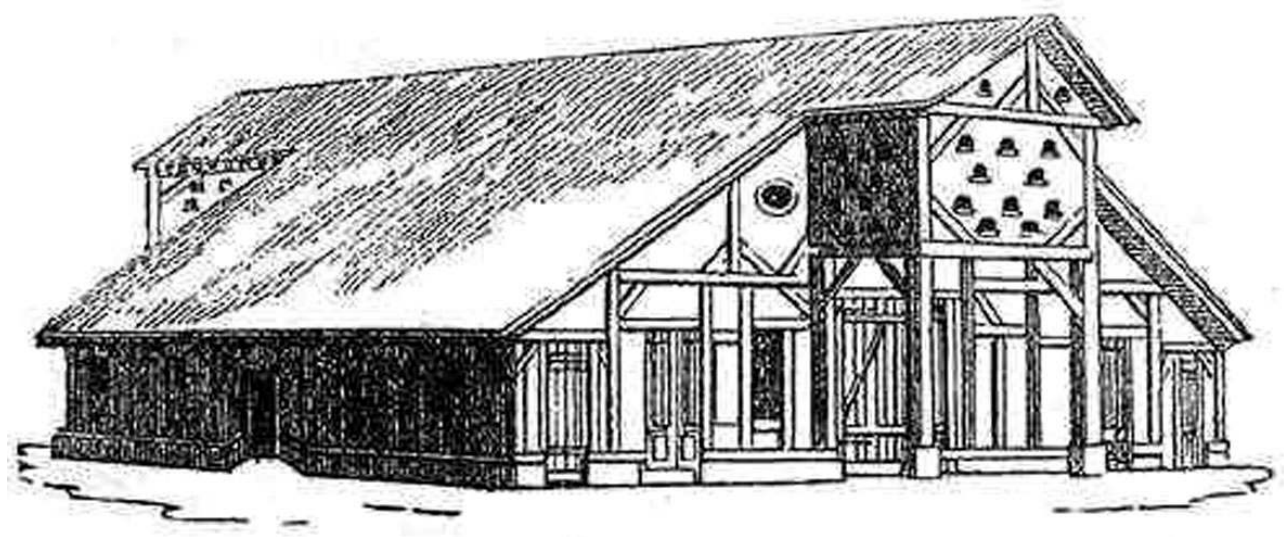

Perspective de la « Grande ferme », d'après Encyclopedia of Cottage, Farm, Villa Architecture, (1833). La grange, au centre, dotée d'une aire à battre le blé et l'avoine est encadrée par une vacherie et une écurie de douze bêtes. Au-delà, dans les parties extérieures, sont situés d'un côté la laiterie, la fromagerie et la loge pour les veaux et de l'autre le poulailler, la sellerie et la porcherie. Le colombier et la volière sont placés au-dessus du porche d'entrée.

Repro. Nègre, Valérie. (c) Valérie Nègre. 
Ces quelques exemples suffisent à montrer que Morel-Vindé ne part pas de «convenances locales» pour concevoir ses modèles. L'orientation et la forme du terrain ne sont pas prises en compte dans la définition du projet. La ferme est orientée selon les points cardinaux et son organisation générale est celle d'une ferme Normande. L'agronome ne porte aucun intérêt aux techniques et aux usages ruraux. C'est un trait qu'il partage avec plusieurs de ses contemporains : "Les ouvriers de la campagne pouvait-on lire dans le Nouveau cours complet d'agriculture de l'abbé Rozier (1821) sont généralement si ignorants et si maladroits, que souvent avec les meilleurs matériaux disponibles, ils ne peuvent parvenir à faire des constructions solides ${ }^{25}$. Morel-Vindé ne reconnaît aux artisans aucune habileté acquise par l'habitude et les futurs usagers de sa ferme sont assimilés aux indigents sans compétences destinés à peupler les colonies hollandaises. Il est clair que dans un tel contexte, rien ne s'oppose à l'importation de techniques ou de types d'édifices radicalement différents : modèles anglais et hollandais pour la construction des gerbiers, modèles russes pour le mode de chauffage. De ce point de vue, les choix de l'agronome ne sont pas originaux, les dessins et les maquettes de constructions rurales déposés en 1840 au Conservatoire des arts et Métiers pour éclairer les constructeurs montrent l'importance des modèles anglais, allemands et suisses ${ }^{26}$. Pour la France, le catalogue de la collection mentionne une "ferme cauchoise», " une étable belge et une étable limousine» et des "ruches angevines $»^{27}$.

13 Comment ces modèles étaient-ils reçus par les habitants des campagnes et des villes ? Si l'on en croit John Claudius Loudon, en Écosse, en Angleterre et dans les régions de construction en pierre en général elles semblaient simples et «sans beauté » (homeliness) : «Un observateur non avisé, écrivait-il, profondément marqué par les préjugés contre les édifices en bois, préjugés courant en Angleterre, en Écosse et dans les régions de construction en pierre, qui ne comprendrait pas que c'est la fin qui doit déterminer les moyens, méprisera la simplicité et le manque de beauté de cette maison ; mais pour nous, qui connaissons tous les détails de ce projet, il nous parfait en son genre $»^{28}$. Il avouait avoir lui-même construit une maison "sur le plan de MorelVindé " qui n'était pas "très appréciée des personnes de la campagne " 29 . Mais le botaniste écossais, fervent admirateur de Morel-Vindé, n'était pas le meilleur témoin pour apprécier cette question. Ses commentaires montrent néanmoins tout l'intérêt qu'il y aurait à écrire une histoire de la réception de ces projets.

\section{NOTES}

1. - C'est un de ses biographes qui l'écrit : « ... profondément convaincu d'appliquer les sciences positives à l'art agricole pour triompher de la routine». BARRAL, Jean Augustin. «Éloge historique de Morel-Vindé ». Journal d'agriculture pratique, t. 1, janvier à juin 1860, p. 12.

2. - Selon son expression. Voir MOREL-VINDÉ, Charles Gilbert, vicomte de.« Troisième et dernière suite des observations sur la monte et sur l'agnelage, suivie de quelques 
considérations générales sur l'encouragement à donner à l'agriculture, par Morel de Vindé ». Mémoires de la Société d'agriculture d'économie rurale et domestique, t. XIX, 1816, p. 181. Morel-Vindé mène ses expériences dans ses fermes de Chichey et de Le Meix (Marne, région Champagne-Ardenne), dans son domaine autour du château de Magnanville (Seine-et-Oise) acheté en 1791 puis, plus près de Paris, dans le domaine du château de la Celle-Saint-Cloud (Yvelines) acheté en 1804. D'après son biographe, " son faire-valoir direct » à la Celle-Saint-Cloud se composait « de 48 hectares en culture et de 16 hectares de près naturels ; il entretenait sur ce petit espace l'équivalent de 120 têtes de gros bétail ». BARRAL, Jean Augustin. "Éloge historique de Morel-Vindé ». Journal d'agriculture pratique, t. 1, janvier à juin 1860, p. 16.

3. - FRENILLY, François Auguste Fauveau de. Souvenirs du baron de Frénilly, pair de France (1768-1828), publiés avec introduction et notes par Arthur Chuquet. Paris : Plon, 1908, p. 198 et 199.

4. - Il hérite de son grand-père maternel Gilbert Paignon-Dijonval, en 1789, d'importantes valeurs mobilières (1 $500000 \mathrm{fr}$, selon un de ses biographes) ainsi qu'une très importante collection en partie conservée à la bibliothèque du Sénat (fonds d'estampes de cartes et de plans notamment) et au Château de La Motte-Tilly (près de Provins).

5. - Joseph Chénier dans son Tableau historique de l'état et des progrès de la littérature française, depuis 1789 écrivait à propos de ses livres «ce sont des œuvres dont le style est plein de grâce et de fraîcheur, et qui respirent une morale pure ", cité par BARRAL, Jean Augustin, op. cit., p. 13.

6. - Morel-Vindé publie dix mémoires sur la race ovine et cinq mémoires sur les assolements à partir de 1799. Il est nommé membre de la Société d'agriculture et correspondant de l'Académie des Sciences pour la section d'« économie rurale » en 1808, puis membre titulaire de l'Institut dans cette même section en 1824 .

7. - BARRAL, Jean Augustin, op. cit., p. 14.

8. - MOREL-VINDÉ, Charles Gilbert, vicomte de. «Observations sur la monte et sur l'agnelage». Mémoires de la Société d'agriculture d'économie rurale et domestique, t. XVI, 1813, p. 382.

9. - L'expression est de GIRARDIN, Jean. Notices biographiques sur MM. de Morel-Vindé, d'Arcet et Mathieu Dombasle. Rouen : Péron, 1845, p. 110.

10. - Morel-Vindé publie trois brochures et deux recueils sur l'architecture rurale: Plan des râteliers de la Celle-Saint-Cloud, (in- $8^{\circ}$, Huzard, 1808). Extrait du Mémoire lu à l'Institut sur une nouvelle manière de construire les gerbiers à toit mobile (in- $8^{\circ}$, Huzard, 1811); Plans et détails d'une nouvelle construction rurale pour servir de grange, Exécutée à la Celle-Saint-Cloud (in- $8^{\circ}$, Huzard, 1813); Plan, coupe, élévation et détails d'une bergerie, exécutée à la Celle-Saint-Cloud (in-fol, Lusson, 1819); Essai sur les constructions rurales économiques (in-fol., Lusson, 1824).

11. - MOREL-VINDÉ, Charles Gilbert, vicomte de. Essai sur les constructions rurales économiques, contenant leurs plans, coupes, élévations, détails et devis, établis aux plus bas prix possibles...les détails de constructions et devis ont été faits, avec l'approbation de l'auteur par A. L. Lusson, Architecte. Paris : Huzard, 1824. In-fol., 34 p., 36 pl.

12. - LOUDON, John Claudius. Encyclopedia of Cottage, Farm, and Villa Architecture. London : Henry G. Bohn, 1833.

13. - L'édifice est publié dans Plans et détails d'une nouvelle construction rurale pour servir de grange, Exécutée à la Celle-Saint-Cloud, près Versailles... Paris : Mme Huzard, 1813. In- $8^{\circ}$, 12 p., 2 pl., dépl. La brochure est extraite du tome LIII des Annales de l'Agriculture française.

14. - Plans et détails d'une nouvelle construction rurale pour servir de grange, Exécutée à la Celle-SaintCloud, près Versailles... Paris : Mme Huzard, 1813. In-8, 12 p., 2 pl., dépl., p. 6. 
15. - Plans et détails d'une nouvelle construction rurale pour servir de grange, Exécutée à la Celle-SaintCloud, près Versailles... Paris : Mme Huzard, 1813. In- $8^{\circ}, 12$ p., 2 pl., dépl., p. 5.

16. - Plans et détails d'une nouvelle construction rurale pour servir de grange, Exécutée à la Celle-SaintCloud, près Versailles... Paris : Mme Huzard, 1813. In-8º 12 p., 2 pl., dépl., p. 5.

17. - MOREL-VINDÉ, Charles Gilbert, vicomte de. Plan, coupe, élévation et détails d'une bergerie, exécutée à la Celle-Saint-Cloud, près Versailles. Paris : A. L. Lusson, 1819, p. 3. La publication in-folio se compose de [6] p. et [4] pl. À propos du rôle de l'architecte, Morel-Vindé précise que «Beaucoup d'agriculteurs ayant désiré se procurer les plans de cette bergerie, le propriétaire a consenti à ce qu'ils fussent levés et publiés par $\mathrm{M}$. Lusson, architecte, demeurant rue Neuve-de-Seine, $\mathrm{n}^{\circ} 79$, à Paris, à qui il a donné avec empressement cette marque de son estime et de sa confiance ", ibid., p. 3. Les planches de ce recueil sont reproduites dans l'Essai sur les constructions rurales économiques (1824).

18. - Morel-Vindé s'adresse directement au maître charpentier Girodet (Chaussée de Bougival) pour réaliser le gerbier.

19. - L'architecte est l'auteur des dessins et du devis et l'éditeur de l'ensemble. Adrien Louis Lusson (La Flèche, 1788- Rome 1864) travaille pour Morel-Vindé, d'abord à la Celle-Saint-Cloud puis à l'hôtel Vindé acheté par Morel-Vindé en 1819. Il en fait l'état des lieux en 1822 et conduit les travaux en 1827.

20. - Morel-Vindé écrivait: «Je n'avais point la pensée d'offrir, comme modèles, les diverses constructions rurales que j'ai conçues et exécutées dans mes exploitations, lorsqu'au commencement de 1819, je fus appelé à faire partie du Conseil d'agriculture près le Ministère de l'intérieur. Au nombre des utiles projets proposés à ce Conseil par son fondateur, celui de défricher nos immenses landes, en y établissant des colonies du genre de celles formées en Hollande, sous le nom de Frederiks-O'ords, échauffa d'abord notre zèle. Le premier objet dont on devait nécessairement s'occuper était la construction la plus rapide et la plus économique de logemens et de bâtimens d'exploitation nécessaires aux colons: on cita mon exemple; on invoqua mon expérience, je cédai à ces instances si respectables pour moi, et je m'engageai à rédiger et publier mes procédés. Malgré l'affligeant abandon de ce beau projet de colonisation, je remplis aujourd'hui l'engagement que je pris alors »: Essai sur les constructions rurales, op. cit., p. i.

21. - Essai sur les constructions rurales, op. cit., p. ij.

22. - « C'est sur cette idée principale et simple, c'est-à-dire sur l'emploi exclusif de bois de 10 à 12 pieds de long sur 6 pouces d'équarrissage au plus, que j'ai conçu d'abord mes bergeries, puis ensuite toutes les autres constructions que j'ai été dans le cas de projeter, faire ou conseiller. J'ai modifié cette idée première de toutes les manières possibles, et le succès a toujours surpassé mes espérances ». Essai sur les constructions rurales, op. cit., p. ij.

23. - « On remarque peut-être qu'il se trouve dans la cuisine de cette habitation un poteau découvert et qui peut paraître gênant. [...] j'observerai que ce poteau correspond à trois autres pareils, qui sont engagés dans les cloisons de distribution, et que ces quatre poteaux sont nécessaires pour conserver le même système général et économique des bois petits et à courtes portées ». "Habitation du petit propriétaire, tenancier, métayer ». Essai sur les constructions rurales, op. cit., p. 7.

24. - Essai sur les constructions rurales, op. cit., p. ij. 
25. - ROZIER, Abbé. Cours complet d'agriculture Théorique, Pratique, ... Paris : librairie d'Éducation et des Sciences et Arts, t. IX, 1821, article « Maçonnerie », p. 252. On peut lire dans le même ouvrage à l'article "Économie, architecture rurale »: " On trouve encore dans les grandes villes d'excellens ouvriers en tous genres, et là les propriétaires n'ont, pour ainsi dire, qu'à choisir entre ceux qui à l'intelligence réunissent la probité la mieux reconnue; mais il n'en est pas de même dans les campagnes éloignées de ces citées. La routine la plus aveugle et l'ignorance la plus crasse sont le partage de ces prétendus ouvriers, et souvent avec les meilleurs matériaux. Ils ne peuvent parvenir à faire un bâtiment solide ». Ibid., t. V, 1821, Article "Économie, architecture rurale », p. 525.

26. - Le catalogue des collections mentionne une " fosse à fumier système saxon ", une «Étable du Wurtemberg ", une "Bergerie Allemande », une "maison d'habitation et d'exploitation d'un petit cultivateur allemand" une "Ferme du canton de Berne ». MORIN, Arthur. Conservatoire des arts et métiers, catalogue des collections. Paris, 1851, p. 167.

27. - Il faut ajouter à cela les maquettes de la ferme de Morel-Vindé, des écuries de Charles Dombasle, des porcheries de Grignon et des « détails pour la construction en pisé ». Ces maquettes ne semblent plus conservées dans les collections. Ibid., p. 167 et 171.

28. - « A superficial observer, deeply imbued with the prejudices common in Britain, and especially in Scotland and other stone countries, against wooden buildings, and not taking into consideration the fitness of means to ends, will be apt to despise the simplicity and homeliness of this farm house; but to us, who have entered into all the details of this Design, it appears perfect of its kind ». LOUDON, John Claudius. The Encyclopedia of Cottage, Farm, Villa Architecture, 1839, voir « The farm house ».

29. - « I have been building a house on Morel-Vindé's plan, given in the Encycopedia. of Arch, wich is not much approved by our country people, but which is, of course, économical »., LOUDON, John Claudius, . The Architectural magazine, t. II, 1835, p. 138.

\section{RÉSUMÉS}

Charles Gilbert de Morel-Vindé partage de nombreux traits de caractère avec ses contemporains. Convaincu que l'agriculture est le principal fondement de toute économie politique et que l'agriculteur est « un industriel forçant la nature vivante à produire abondamment et au plus bas prix possible», il fait partie de ces nombreux propriétaires agronomes qui appliquent les sciences positives à l'art agricole. Le personnage est intéressant en ce que l'importance de sa fortune et sa position sociale éminente lui permettent de transformer ses propriétés « en fermes d'expériences et d'exemples " et de modéliser de nouveaux types de bâtiments ruraux. Ses publications méritent l'attention car elles permettent d'observer le développement de modèles dont la forme et la localisation sont déterminées par une rationalité indépendante de toute relation avec le lieu, autrement dit des édifices qui ne tiennent pas compte des caractéristiques environnementales, culturelles et identitaires. C'est l'émergence de ce phénomène que l'on 
qualifierait aujourd'hui de « déterritorialisation » que cet article se propose d'examiner à travers quelques projets de l'agronome.

Charles Gilbert de Morel-Vindé shares many of the characteristics of his contemporaries. Convinced that agriculture is the founding principle of all political economy and that the farmer is "an industrialist who forces living nature to produce abundantly at the lowest possible cost", he is one of a large number of land-owning agronomists who apply positive science to the art of agriculture. He is an interesting character in that the extent of his wealth and his eminent social position allow him to transform his properties "into experimental and exemplary farms" and to establish new models for rural buildings. His publications deserve our attention as they allow us to observe the development of models whose form and placement are determined by a logic which is independent of any relationship with their surroundings, in other words, buildings which do not take characteristics related to environment, culture and identity into account. It is the emergence of this phenomenon which would be classified nowadays as "de-territorialisation" that we propose to examine in this article in the light of some of the agronomist's projects.

\section{AUTEUR}

\section{VALÉRIE NÈGRE}

École nationale supérieure d'architecture Paris La Villette et Conservatoire national des arts et métiers (HTTP-CDHTE) valerie-negre@wanadoo.fr 